\title{
MORE ON RINGS ON RINGS ${ }^{1}$
}

\author{
BY IAN RICHARDS
}

Communicated by Gian-Carlo Rota, March 11, 1968

This note is a sequel to the paper On rings on rings by Anatole Beck [1]. The problem we consider (originally proposed by Paul Rosenbloom) is that of characterizing the parameter $\rho$ for the annulus $\Omega=\{1<|z|<\rho\}$ in terms of the ring $R$ of bounded analytic functions on this annulus. Beck's solution involves properties of univalent functions, and although the subset of univalent functions in $R$ can be characterized algebraically, it seems preferable to avoid this complication.

THEOREM. Let $R$ be the Banach algebra of all bounded analytic functions on the annulus $\Omega=\{1<|z|<\rho\}$ endowed with the usual sup norm. Let $U$ be the set of invertible elements in $R$ (i.e., the set of $f \in R$ for which $1 / f \in R)$, and let $H$ be the set of $f \in R$ which possess nth roots $f^{1 / n} \in R$ for all $n$. Then

$$
\rho=\inf _{f \in U-B}\|f\| \cdot\left\|f^{-1}\right\| .
$$

Proof. The proof is based on a theorem of Schiffer and Huber (cf. [2]):

Let $f: \Omega \rightarrow \Omega$ be analytic and map a generator $\gamma$ for the homology group $H_{1}(\Omega)$ onto a curve which is homologous to $\gamma^{q}$. Then $q=0,1$, or -1 , and in the last two cases $f(z)$ is a constant multiple of $z$ or $1 / z$ respectively.

To deduce our result, we note first that $f(z)=z$ satisfies $\|f\| \cdot\left\|f^{-1}\right\|=\rho$. Now suppose that $f$ is an element of $U-H$ such that $\|f\| \cdot\left\|f^{-1}\right\|<\rho$. Multiplying $f$ by a constant, we can adjust the norms so that $\|f\|<\rho$ and $\left\|f^{-1}\right\|<1$. Then $f$ maps the annulus $\{1<|z|<\rho\}$ into itself. Since $f \notin H, f^{1 / n}$ fails to exist for some $n$, and hence if $\gamma$ is a generator for $H_{1}(\Omega), f(\gamma)$ cannot be homologous to zero. Thus by the SchifferHuber theorem, $f(z)$ is a constant multiple of either $z$ or $1 / z$; in either case $\|f\| \cdot\left\|f^{-1}\right\|=\rho$.

\section{REFERENCES}

1. A. Beck, On rings on rings, Proc. Amer. Math Soc. 15 (1964), 350-353.

2. E. Reich, Elementary proof of a theorem on conformal rigidity, Proc. Amer. Math. Soc. 17 (1966), 644-645.

UNIVERSITY OF MINNESOTA

1 Partially supported by NSF Grant GP-4033. 